\title{
La dévotion à sainte Anne de Beaupré : éléments de réflexion sur une dévotion liée à un centre de pèlerinage
}

\section{Anne Doran-Jacques}

Volume 50, numéro 1, 1983

Bilan de l'histoire religieuse au Canada

Canadian Catholic History: A survey

URI : https://id.erudit.org/iderudit/1007047ar

DOI : https://doi.org/10.7202/1007047ar

Aller au sommaire du numéro

Éditeur(s)

Les Éditions Historia Ecclesiæ Catholicæ Canadensis Inc.

ISSN

0318-6172 (imprimé)

1927-7067 (numérique)

Découvrir la revue

Citer cet article

Doran-Jacques, A. (1983). La dévotion à sainte Anne de Beaupré : éléments de réflexion sur une dévotion liée à un centre de pèlerinage. Sessions d'étude Société canadienne d'histoire de l'Église catholique, 50(1), 281-306.

https://doi.org/10.7202/1007047ar

Tous droits réservés @ Les Éditions Historia Ecclesiæ Catholicæ Canadensis Inc., 1983
Ce document est protégé par la loi sur le droit d'auteur. L'utilisation des services d'Érudit (y compris la reproduction) est assujettie à sa politique d'utilisation que vous pouvez consulter en ligne. 


\section{La dévotion à sainte Anne de Beaupré:}

\section{éléments de réflexion sur une dévotion liée \\ à un centre de pèlerinage}

En posant le titre: la dévotion à sainte Anne de Beaupré, on énonce déjà un paradoxe. En effet, ce titre contient deux propositions: la dévotion à sainte Anne, réalité qui de soi semble complète, et un complément qui semble superflu: Beaupré. Un terme qui fait référence à l'universel en ce qu'il représente une personne sainte, la mère de Marie et la grand-mère du Christ, reconnue comme telle par tous ceux qui la connaissent; un autre lié à un lieu qui particularise le culte en liant une image universelle aux conditions particulières d'un lieu géographique. Et cela est tellement réellement contradictoire qu'au delà d'une réaction de pèlerin du type: «J'avoue que j'ai bien confiance à sainte Anne, qu'on prie également en France, à Auray. Je suis venue au Canada en touriste, mais à Sainte-Anne de Beaupré en pèlerine” ', qui reconnaît l'universalité de la dévotion au delà d'une localisatịon particulière, nous pouvons aussi bien arriver à cette sorte d'esquisse caricaturale de la situation pèlerine: "Je n'ai aucune confiance en sainte Anne de Beaupré. Mais j'ai pleinement confiance en sainte Anne d'Auray" ${ }^{2}$. On pose ici la contradiction: la même sainte Anne, mais s'agit-il vraiment de la même, suscite une réaction affective vécue positivement dans un lieu, négativement dans l'autre. Comment est-ce possible et qu'est-ce que cette sorte de situation limite concernant une dévotion liée à un centre de pèlerinage peut nous suggérer sur la réalité à la fois universelle et particulière qu'on y vit?

Évidemment, il nous faut remarquer que cette contradiction n'est pas vécue comme telle par le pèlerin ordinaire qui ne connaît d'expérience que sainte Anne de Beaupré ou plutôt qui a bien des chances de connaître en plus de ce grand centre de référence un

\footnotetext{
${ }^{1}$ Professeur de France - 70 ans.

${ }^{2}$ Institutrice de Paris - 50 ans.
} 
petit sanctuaire local de pèlerinage à sainte Anne, sanctuaire familier et souvent d'usage habituel. Ici le rapport du petit centre au grand en est un de continuité : le petit centre met à la disposition du "dévot" un lieu d'assouvissement de sa dévotion vécue en relation avec le grand centre. Et la multiplicité des petits centres révèle le besoin d'avoir une sainte Anne proche. Mais cette Sainte-Anne à portée de main, d'usage régulier pourrait-on dire, est vécue en référence au lieu par excellence de sainte Anne, celui de Beaupré. Ici aussi le culte est donc vécu dans sa liaison à un lieu qui le particularise, celui du grand centre.

Si nous reprenons les termes mêmes de notre proposition: la dévotion à sainte Anne de Beaupré, qu'y trouve-nous? L'attribution d'une figure sainte à un lieu. Et c'est même la soudure parfaite entre ces deux termes qui aboutit à cette caricature que nous évoquions plus haut: le rapport du saint à son lieu est si indissoluble que le même saint vénéré à un autre lieu est sans rapport avec lui. En attachant le saint à des coordonnées spatio-temporelles, le lieu semble donc avoir tendance à particulariser le saint. C'est une étude de ce rapport entre le saint et son lieu que nous voudrions entreprendre ici, mais à partir du vécu des pèlerins de Sainte-Anne de Beaupré. $\mathrm{Si}$ la contradiction entre le global et le particulier a des chances d'être résolue, c'est bien ici, dans le vécu, qu'elle doit l'être. Pour cela, nous allons nous référer à différents types de témoignages de pèlerins, feuillets de prière déposés au pied de la statue dans l'immédiat de la célébration de la fête de sainte Anne, entrevues de pèlerins et récits de faveurs rapportées au bureau du sanctuaire de 1977 à $1982^{3}$. Qu'est-ce que ces documents ont à nous apprendre sur la dévotion à sainte Anne de Beaupré, sur la dévotion à cette sainte vécue en ce lieu particulier?

\footnotetext{
${ }^{3} S$ 'il faut rappeler les coordonnées essentielles du pèlerinage, disons que Ste-Anne de Beaupré, dont on estimait dans les livrets touristiques publiés par le gouvernement du Québec en 1972-73 qu'il attirait à chaque année son million de visiteurs, est un centre de pèlerinage qui a plus de 300 ans, 325 ans en 1983 pour être précis. Le légendaire attribue sa fondation à un voeu fait par des matelots pris dans une tempête au Cap Tourmente de bâtir une chapelle à sainte Anne sur le rivage s'ils étaient sauvés. Dans les faits, les premiers documents remontent à 1658 , au moment où un habitant de la Côte de Beaupré, Étienne de Lessard, donna une partie de sa terre pour la construction d'une église ou chapelle.
} 


\section{LES FEUILLETS DE PRIÈRE}

Les premiers documents de notre analyse que nous nommons feuillets de prière ont été recueillis dans des boîtes placées à cet effet au pied de la statue, aux environs immédiats de la fête de cet été (26 juillet 1983). Le pèlerinage fournit en effet des feuillets intitulés: "Ma supplique à sainte Anne - My petition to Saint Anne", de petit format et destinés à recevoir les intentions des pèlerins. La plupart des intentions seront rédigées sur ces feuillets alors que d'autres s'inscriront sur toutes sortes de papiers depuis la page arrachée du calepin jusqu'au morceau d'une feuille de publicité quelconque recueillie en route. Enfin on trouve quelques véritables lettres et ce phénomène semble lié à la célébration de la fête; on trouvera d'ailleurs dans les feuillets un certain nombre de "bonne fête sainte Anne" ou des allusions à une démarche sur les lieux du pèlerinage liée à cet événement.

Une étude antérieure ${ }^{4}$ des feuillets de prière nous faisait apparaître la globalité comme premier caractère des demandes faites à Ste-Anne de Beaupré. Intentions concernant la santé, l'organisation de la vie concrète, travail, courage dans les situations difficiles mais aussi intentions spirituelles et notamment une demande de conversion. Nous concluions à ce moment-là que la demande faite à Ste-Anne était une demande où vie matérielle, à commencer par la santé du corps, et démarche spirituelle trouvaient leur expression et se conjugaient dans une unité qui laissait place à l'un et à l'autre et s'exprimait particulièrement dans la demande de paix.

Nous trouvons encore ici beaucoup de demandes qui présentent ce même caractère de globalité. Ainsi: «Je demande la santé du corps et de l'esprit. Conversion de tous mes enfants. Garde-les dans la foi et l'amour de ton petit fils bonne Ste Anne. Merci - la santé de l'ouvrage pour $\mathrm{Nf}^{5}$ et la vente de son auto - la paix dans tous les foyers"; "Je demande la santé pour $\mathrm{Nf}$. Je sollicite pour $\mathrm{Nf}$ le sacrement de mariage. Je demande du travail pour mes neveux. Enfin je confie ma famille à la Bonne Ste-Anne». On fait ici le tour de son environnement familier,

\footnotetext{
${ }^{4}$ Il s'agit d'une thèse portant sur Le pèlerinage à Sainte-Anne de Beaupré; L'actuel 1958-1973 déposée à l'École des Hautes Études en Sciences Sociales (Paris, 1979, 3 v., $814 \mathrm{f}$ ) et dont on peut aussi trouver un exemplaire à la bibliothèque de l'Université du Québec à Chicoutimi.

${ }^{5}$ Nous désignons par $\mathrm{Nf}$ un nom féminin et par $\mathrm{Mn}$ un nom masculin que nous rencontrons dans le texte.
} 
famille, proches et on énumère les besoins matériels, spirituels, puisque les deux demandes relèvent de ce soutien de tout l'être qu'on vient chercher.

Pourtant à l'occasion la demande pourra se faire plus poignante et deviendra unique: "Que la paix revienne dans notre ménage»; "Je désire que mon garçon arrête de boire"; "Dearest Saint Anne please bring back Nf, Nf \& Nf back to your son, let them love you also, our Blessed Mother, as they used to. Make them understand \& let them love your Grandson, bring them back to the Church. Love». Ici le pèlerinage relève plus du panique, l'intention mobilise toute la prière.

Nous avons donc affaire à deux types de demandes: demande à caractère global de celui qui maintient une relation de longue haleine avec la sainte et qui énumère les besoins de ses proches. Demande en situation d'urgence où la prière exprime le besoin qui monopolise toute l'attention et qui peut-être a déterminé le pèlerinage. Appartenant à la première catégorie, de loin la plus importante, nous trouvons aussi un type d'expression neuf où cherche à s'exprimer un vocabulaire théologique plus développé en relation semble-t-il avec une nouvelle pastorale et certains mouvements ou groupes de prière. On mentionne plus souvent l'Esprit Saint, on cherche à expliciter une hiérarchie dans l'objet de la demande. Ainsi:

\begin{abstract}
Bonne sainte Anne - ma grande soeur - ma grande ami - ma protectrice dans mes jeunes années - Celle qui a guéri papa - tu t'en souviens Benis sois loué ton Petit fils Jesus - notre Sauveur de t'avoir donné à nous - comme grand-mère - je te demande sainte Anne de protéger mon mari - mes enfants - tous ceux que nous portons dans notre coeur - et de faire en sorte la volonté du Père s'accomplisse en nous.
\end{abstract}

Bonne Sainte Anne - va dire à ton petit-fils Jésus - de toucher le coeur de $\mathrm{Nm}$ - Veux-tu? Que ta fille Marie le prenne dans ses bras! et que son coeur de pierre soit transformé en coeur de chair par le dynamisme et la force de l'Esprit-Saint Merci Anne! Je t'embrasse bien fort:

Ou encore: «Suppliez le Père d'enlever tout ce qui est faux dans notre coeur pour mieux découvrir Jésus à $\mathrm{Nm}$ et $\mathrm{Nf}$. Enlevez dans son corps toute maladie pour $\mathrm{Nm}$. Et que $\mathrm{Nm}$ découvre l'amour du Père. Merci pour les merveilles dans les oreilles à Nm». Ici la demande est souvent formulée plus longuement, avec une insistance plus marquée pour les intentions à caractère spirituel mais elle 
relève de cette même globalité à laquelle nous faisions allusion plus haut: on évoque encore ici l'ensemble des besoins corporels et spirituels des proches. S'il y a formulation nouvelle donc, le fond reste essentiellement le même; on présente chacun à sainte Anne en évoquant ce qui peut être amélioré.

L'ensemble des demandes nous ouvre deux voies à approfondir: la relation avec sainte Anne à qui on fait toutes ces demandes et le nom qu'on lui donne ou qu'on se donne dans sa relation à elle; la volonté d'un mieux-être qu'expriment les demandes.

Essayons dans un premier temps de mieux cerner cette relation avec la sainte qu'expriment les feuillets. La première évidence qui caractérise cette relation est qu'il s'agit d'une relation affective. Voici deux exemples entre cent autres :

O ma belle bonne Sainte Anne je t'aime de tout mon coeur et je te souhaite bonne fête Je te demande la conversion de mes enfants je les aiment et toi aussi je sais que tu les aimes Je vous demande la guerison de mas jambes - pour que je puisse à la messe priez.

Et:

Bonne Fête Ste-Anne. Je viens encore implorer tes bienfaits. Fasse que mon mari retrouve sa joie de vivre et que ma famille soit heureuse à nouveau. Permets à mes enfants de travailler, tu connais leurs besoins. O Bonne Ste-Anne, jamais je ne pourrai oublier ce que tu as fait pour moi. J'ai confiance en toi, et je t'en supplie par ton petit-Fils Jesus augmente en moi la foi. Merci pour mon beau-frère et sa famille, ne les oublie pas. Je te confie aussi mes frères \& mes soeurs. Et si cela est possible, je reviendrai te voir ô Bonne Sainte-Anne que j'aime tant. Au revoir et Bonne Fête.

Bel exemple de globalité encore une fois, mais dont l'expression plus abondante permet de saisir tout le caractère affectif: on vient souhaiter bonne fête à quelqu'un qu'on aime et avec qui on est en relation et ce souhait ouvre et termine la prière; on évoque les bienfaits passés de sainte Anne qui ont créé un lien qui ne s'effacera pas. On demande à nouveau en affirmant sa confiance. En fait, on peut saisir ici le processus de cet échange affectif: on aime la sainte, on affirme sa confiance et cette affirmation constitue en quelque sorte un don de soi dont on attend une réponse dans l'obtention de ce qui est demandé. Dans la mesure même où l'on aime et où l'on a confiance, on se 
confie à sainte Anne, et le vocabulaire qui peut être très fort ${ }^{6}$ indique véritablement un don de soi, un engagement de toute la personne envers la sainte dans l'expression du «j'ai confiance». En voici un exemple très touchant dans sa simplicité expressive:

Merci Bonne St Anne de m'avoir guérir de ma gorge Je vous demande toujours quelques chose mais j'ai temps confiance en vous. Là ses mains qu'elles me font mal je le sais que vous aller faire de quoi Donnez moi l'amour aussi envers mon mari je ne sais pas des fois je pense qu'il ne aime plus en tout cas je ne vous laisse pas j'ai trop confiance Aussi aidez mes enfants surtout $\mathrm{Nf}$ dans ses études et amour Faites de $\mathrm{Nm}$ soit heureux. Que $\mathrm{Nm}$ soit bien. Aidez à tous.

Cette relation avec la sainte en est une d'ouverture de l'être dans un mouvement de confiance. Cet amour, cette confiance, ce don de soi dans la relation doivent être propres à mouvoir l'action de la sainte. Ainsi cette intention formulée par un enfant qui, dans la simplicité de sa formulation, démontre bien le mécanisme de l'échange: "Ma bonne sainte Anne Je suis content de venir vous voir et j'aurais une faveur importante à vous demande $S$ 'il vous plait gueriser mon grand père qui est paralyser présentement s'il vous plait je vous en prit car je vou aime beaucoup merci». Il y a lien attendu de cause à effet entre le «je vous en prit" et le "car je vous aime». D'une façon moins enfantine, mais qui n'est pas étrangère à la précédente, nous retrouvons aussi :

Holy St. Anne, beloved grand mother of Jesus look with pity on my grandson $\mathrm{Nm}$ and Heal him. Let not my faith and trust weaken at a time like this when he seems to worsen in his attitude towards his parents and only brother.

With all fervour I shall be kneeling as I drop this petition at your feet and wait for you to work the miracle we are all looking forward to through the merciful Look of Jesus.

Grant us all at home Peace - \& I promise to remain forever your faithful.

July 26th, 1983.

\footnotetext{
${ }^{6}$ Nous trouvons notamment quelques exemples de l'expression «Prenez-le dans vos bras»: «Nf qui est malade alcoolique je te la donne prend la dans tes bras - présente la à Dieu Notre Père». Ou encore: «Faite quelque chose pour ma $\mathrm{Nf}$, selon la volonté de Dieu. Qu'elle guerisse ou prenez là dans vos bras - je vous en prie - Quel vie pour elle, elle n'a pas conscience de cette belle vie, soulagez ses souffrances mentales, toujours selon la volonté de Dieu w.
} 
Ici, l'objet même de la promesse qu'on fait pour obtenir la faveur désirée est la fidélité d'une relation qui engage la personne envers la sainte. Le don consiste en cette sorte d' "amitié sacrée" qui lie le pèlerin à la suite et lui ouvre l'accès à ses faveurs.

Cette relation vécue sous un mode éminemment affectif et qui engage la personne dans une relation d'amour envers la sainte peutelle être précisée encore? Oui, si nous considérons les titres donnés à sainte Anne ou la façon de qualifier le pèlerin dans sa relation à elle. Le titre est attribué à sainte Anne est double: on la nomme tantôt mère et tantôt grand-mère. En fait cette ambiguitté vient de ce qu'elle est à la fois mère et grand-mère et qu'on s'adresse à elle à ce double niveau de sa maternité:

Bonne Ste-Anne

Bonjour

je viens te prier Je suis grand maman comme vous ! et mère...

De la même manière, le pèlerin se dira tantôt enfant et tantôt petit-fils ou petite-fille de sainte Anne et même, expression la plus claire de son double rôle, les deux à la fois comme dans cette prière qui se présente sous forme double et qui est signée dans un cas: "Your grand Child" et dans l'autre "Your child". Double rôle de mère et de grand-mère qui détermine vis-à-vis elle une double situation du pèlerin à la fois enfant et petit-enfant de la sainte.

Toutes les citations que nous venons de faire de textes de la prière pèlerine demandent sous une forme ou une autre, à l'un ou l'autre des niveaux de l'être, l'avènement d'un mieux ou d'un bien. Cette recherche d'un mieux qui dans certains cas va se traduire par une nette volonté de changement, par un besoin de renouvellement de tout l'être est caractéristique de la prière à sainte Anne. Ainsi: «J'ai un besoin que tout change dans ma vie et je compte sur vous». Et:

Grand-maman d'amour, c'est un appel au secours que je te lance. C'est un cri de détresse. Je viens à toi le coeur en larmes. Je suis malade grand-maman... Je suis tannée grand-maman d'être dans cet état. Demande au Seigneur de refaire tout mon être, de me redonner la force, une meilleure santé. Qu'il fasse grandir ma foi. qu'Il me rende un témoin joyeux de lui. Qu'Il m'aide dans mon travail scolaire. 
ou encore:

Bonne Sainte Anne je vous demande de me donner du courage parce que j'ai tellement de difficultés à supporté ce qui m'arrive aidez-moi à me prendre en main je suis une fille très complexe aidez-moi a vivre une vie remplie pas juste de découragement mais de fun aidez-moi a me remonter le morale et a remonter la pente de ma vie.

Et cette autre qui vient témoigner de son désarroi et de désir confus d'un renouveau:

\section{Bonne sainte Anne}

Je mappel Nf Je suit une Drogué et une hommosexuelle aide-moi à vivre l'amour dans la joie et le bonheure. J'ai besoin d'être conprie, et je veux croire en toi. J'ai 19 ans et je suit perdu. Je t'aime

Samedi 23 juillet 1983

Les feuillets nous apprennent donc beaucoup sur la relation qui existe entre les pèlerins et la sainte: relation affective qui lie à une sainte qu'il considère doublement comme sa mère le pèlerin qui s'offre à elle dans un mouvement de confiance dont il attend une réponse l'établissant lui ou ses proches dans un plus-être qui l'atteint tant à un niveau physique que spirituel. Ils ne nous livrent pourtant à peu près rien sur le lieu. Faudrait-il conclure que le lieu joue un rôle superflu dans cette relation pèlerin-figure sainte? On ne parle pas du lieu ici, on le vit. Si les feuillets existent, c'est au lieu qu'on le doit. Sinon, que pourrait-on trouver d'autre qu'une prière dans le secret des coeurs? La prière écrite sur les feuillets suppose un double besoin du lieu: besoin du pèlerinage d'abord, donc déplacement au lieu, et ensuite besoin de laisser une marque tangible de sa prière, de la prolonger en quelque sorte, de l'inscrire de façon palpable lui aussi. Et complémentaire de ce besoin, celui que nous avons retrouvé dans quelques cas, d'apporter une photo au verso de laquelle on a rédigé des intentions. Loin de dévaloriser l'action du lieu dans la prière pèlerine, la prière des feuillets en dévoile plutôt le besoin essentiel. 


\section{LES ENTREVUES DE PÈLERINS}

Pourtant il est un autre matériau qui se révèle beaucoup plus prolixe dans son discours sur le lieu: les entrevues des pèlerins ${ }^{7}$. Ici, suscité par les questions des enquêteurs, le discours sur le lieu abonde.

Une des premières évidences qui ressort du témoignage pèlerin est que ce lieu est un lieu où l'on prie. La réponse revient si souvent et si spontanément que nous touchons sûrement là une des réalités premières du pèlerinage :

Je ne sais ce qu'il y a ici, mais le temps passe plus vite qu'ailleurs. Il y a de la paix, de la sérénité; on a toujours envie de prier. (Organiste de Brooklyn - 63 ans)

Ce que j'aime ici c'est qu'on peut faire l'expérience vivante de l'Église qui prie et qui croit. De nos jours ce n'est pas facile de vivre sa foi... On entre ici et «ouf!», on se dirait dans un autre monde. La prière est la vie de tout le monde et tout semble normal de prier. (Étudiant de la Petite-Rivière-Saint-François - 17 ans)

C'est la première fois de ma vie que je prie à mon goût dans un chemin de croix. (Laitier de Pont-Rouge)

Je viens depuis trois ans, mais je viens pour prier. La neuvaine, c'est un beau temps pour prier. (Handicapé de Springfield, 27 ans)

Vous ne réalisez probablement pas vous autres qui vivez ici la paix et la tranquillité qu'on y trouve. Ca nous saisit en arrivant... C'est très spécial et on se sent comme chez soi. On peut prier ensemble mais il y a de grandes nappes de silence où l'on peut prier tant qu'on veut. On n'est pas achalé pour des riens... pas de solliciteurs non plus. On prie et on dirait qu'on ne s'en aperçoit même pas. (Usinier d'Hartford)

\footnotetext{
${ }^{7}$ Ces entrevues ont été réalisées par un groupe d'hôtes et d'hôtesses travaillant sur les terrains du sanctuaire et qui avaient entre autres pour mission de poser quelques questions aux pèlerins portant sur leurs motivations, leurs impressions, la célébration qu'ils avaient préférée, leur perception de la sainte, etc. À voir la diversité et le contenu spontané des réponses, on a bien l'impression que les questions n'ont fait que servir de cadre à l'expression pèlerine. Ces entrevues sont publiées dans le Rapport sur les activités pastorales au sanctuaire de Sainte-Anne de Beaupré pour les saisons 1977-1978-19791980-1981-1982, rédigé en collaboration et publié par le Conseil du Sanctuaire. Ces mêmes sources nous donnent aussi le texte des récits de faveurs rapportées au Bureau du sanctuaire que nous étudions en troisème partie de cette analyse.
} 
C'est un endroit où l'on prie, où il devient naturel de prier. C'est un endroit qui nous établit dans la prière. C'est un endroit qui élargit la prière aux dimensions du monde ( Moi je fréquente une petite église de banlieue; on a tout juste ce qu'il faut pour célébrer. Quand j'ai vu commencer la célébration dans cette église si vaste, avec de la belle lumière à pleines verrières et des chants qui se répercutaient dans la voûte j'avais l'impression que ma prière s'élargissait aux dimensions du monde" - Dentiste de Long Island), sans doute à cause de cette dimension collective qu'elle lui confere mạis peut-être surtout à cause de cette unité supérieure d'un peuple priant qui se crée ici: «La liturgie ici prend un caractère qu'on ne lui trouve pas dans nos paroisses. C'est plus qu'une foule... c'est comme un peuple qui prie» (Dame de SteAgathe, Maine).

Un dernier témoignage enfin reprend les éléments de presque tous les autres (on est pris dans la prière, diversité de la foule qui trouve son unité dans la prière dite ou chantée, abolition des barrières sociales que nous allons retrouver à l'instant) en ajoutant une dimension qui n'était pas apparue jusqu'ici': le rôle de la nature dans les célébrations accomplies au lieu du pèlerinage:

La procession aux flambeaux, je l'ai faite sur le perron. Trop lâche pour marcher. Mais je me suis fait prendre: j'ai prié là en m..., comme j'avais jamais fait depuis longtemps. Du monde, du monde, des jeunes, des vieux, des gens qui se traînent la patte... et tout le monde chante, prie, semble aux petits oiseaux. Personne ne pense à ce qu'on va dire d'eux; la procession aux flambeaux, c'est la plus belle patente qu'il $\mathrm{y}$ a à Ste-Anne pour surprendre le monde. Faut dire que tout y était: une température douce et belle... un petit vent ou plutôt une brise, qui était comme une caresse et surtout la lune dans son plein. Je m'en cache pas, j'ai braillé comme un veau. Un vrai veau! Peu importe ce que les gens ont pensé autour de moi, je ne connaissais personne. Même si je les avais connus, ce soir-là, j'étais trop navré pour me retenir. (Forgeron de la Rive sud, 50 ans )

Nous pourrions reprendre un à un les différents éléments de ces témoignages et les confirmer par d'autres. Le lieu du pèlerinage est un lieu de paix, de silence, de repos, un lieu où on se sent chez 
soi, un lieu qui abolit les barrières sociales ${ }^{8}$, un lieu où l'évidence de la prière et de la foi constituent un témoignage.

Nous venons de voir comment le lieu nous établit de plain-pied et comme de soi dans la prière. Cela nous amène maintenant à approfondir une réalité complémentaire de celle-ci : ce lieu nous amène à Dieu :

On est sans cesse ramené à Dieu, mais simplement: prières à sainte Anne, atmosphère, tout ce qu'on a sous les yeux, contact avec les prêtres... Tout ça mis ensemble, nous secoue fortement à l'intérieur. Et il n'y a rien de forcé ou de factice... C'est tout simplement que cela se produit, presque malgré nous autres. (Dame de Philadelphie)

Et puisqu'il faut lâcher le mot, cette orientation vers Dieu est souvent évoquée par les pèlerins comme une conversion. On vient pour se réconcilier avec Dieu ou bien pris par ce qui se passe sur les lieux, on revient à lui :

Nous sommes venus pour voir le site où avaient vécu nos ancêtres. Mais nous avons vu les malades, la messe, la procession, les pèlerins qui prient tout le temps. Moi qui ne pratiquais plus depuis des années, je n'ai pu tenir le coup: je suis allé me confesser, je suis allé à la messe, j'ai communié et j'assiste à toutes les messes que je puis pendant le temps que je suis ici. (Professeur du Maine, 25 ans $)^{9}$

Cette conversion peut prendre la forme d'un changement de vie. Changement de vie recherché et qui vient s'appuyer sur une démarche pèlerine: "Je suis alcoolique et célibataire et je travaille sur les bateaux.

\footnotetext{
${ }^{8}$ Et le témoignage des malades là-dessus est très clair : « Je prie beaucoup pour avoir du courage. C'est aussi une vacance de l'esprit. Vous ne savez pas ce que c'est que d'être enfermé entre quatre murs tout le temps, avec tout du monde malade et éclopé. Vous ne pouvez imaginer que cela représente pour moi de voir du monde normal (Handicapé de la région de Québec - 30 ans). Une autre dira plus encore: le pèlerinage lui permet de poser le même geste que tout le monde et c'est en cela qu'il abolit les différences: «Je suis enfermée toute l'année dans une résidence pour handicapés. Ici, on peut prier et il y a beaucoup de gens. C'est un geste que je peux poser comme les autres et je ne suis pas différentes des autres parce que je pose un geste comme celui-là. Je me retrouve ici» (Handicapée de Montréal - 40 ans). Mais les gens «normaux» aussi vivent l'abolition de ce qui marque la différence entre les hommes. Comment constituer un peuple qui prie et vivre ce qui nous sépare?

${ }^{9}$ Remarquons que le point de départ de cette conversion se situait dans une volonté de garder un lien avec ses racines et un passé vécu dans la foi. Le rôle de cet élément traditionnel est une constante des chants.
} 
Je viens d'arrêter de boire. J'ai fait des promesses à sainte Anne; il faut qu'elle me donne un coup de main" (Homme de Boston, 51 ans). Nouvelle orientation de vie consécutive à une démarche pèlerine: "Je n'ai pas été guéri physiquement, mais je me suis débarrassé ici d'un problème de boisson. C'est en regardant les béquilles que je me suis fait une raison. Il y a des béquilles morales qui sont plus lourdes à porter que des béquilles physiques" (Postier de Montréal). Réflexion sur sa vie pour bien l'orienter, en particulier dans les cas où on envisage la séparation ou le divorce, dans les cas où cette visite demeure l'ultime recours avant une rupture.

Si ce lieu engage à un approfondissement du spirituel, rend à l'homme sa capacité de prier ou l'oriente vers une conversion, si ce lieu donc mène à un plus être spirituel, il est aussi, faut-il le rappeler, voie vers une positivité qui se manifeste au plan physique. Nous n'analyserons pas maintenant tous les témoignages de gens qui viennent parce qu'ils ont déjà obtenu une guérison, qui viennent en demander une, qui connaissent quelque'un qui a été guéri en ce lieu. Ne retenons qu'une chose pour le moment: le "miracle" est une réalité bien vivante à Sainte-Anne.

Je ne sais si vous êtes frappés comme moi de voir comment, dans les effets du lieu sur le pèlerin, on retrouve la même orientation double qu'au niveau de cette globalité que nous avions remarquée dans la prière pèlerine: intentions concernant un mieux-être du corps ou de l'âme, puisque pour distinguer il nous faut employer les termes d'un langage dualiste. Il y a positivité des effets de la démarche pèlerine à la fois dans un approfondissement spirituel et dans un retour à la santé du corps. En fait, le pèlerin lui, ne distingue pas: à chaque fois qu'il y a besoin, sainte Anne est là, quel que soit ce besoin:

Sainte Anne me voit arriver à ses pieds chaque fois que j'ai à faire face à un problème important. Présentement je traverse une épreuve très forte et je viens lui demander de m'en sortir. (Dame de Québec - 30 ans )

Tout blocage, à quelque niveau de l'être, dans le cheminement positif de la vie, intéresse sainte Anne. Mais on peut pousser plus loin l'analyse: c'est dans une positivité qui rejoint tout l'être que s'accomplira le miracle ou la faveur; la distinction guérison physique et guérison spirituelle n'a plus de sens puisqu'il s'agit de faire évoluer l'être tout entier vers un plus et qu'à ce niveau le physique sera lié au spirituel: 
On fait bien de venir à Ste-Anne pour obtenir une guérison. J'ai été guéri d'une maladie de coeur il y a quelques années; même plus de trace maintenant. C'est la foi qui est à l'origine de ce rendez-vous: guérison externe ou changement intérieur, tout cela procède d'une démarche de foi. (Comptable du Niagara, 55 ans)

L'établissement de l'être dans un renouveau de positivité s'exprimera souvent aussi, particulièrement dans le cas de grands malades et d'handicapés, par une demande ou une obtention de courage qu'on nomme comme tel le plus souvent:

Ça fait longtemps que je suis malade. Je ne viens pas demander ma guérison. Je viens simplement offrir ma maladie à sainte Anne. Je viens lui demander le courage qu'il me faut. (Dame du Nouveau-Brunswick)

mais aussi «apprendre à vivre»,

J'ai eu un accident très grave en travaillant à la construction; je suis resté invalide jusqu'à la fin de mes jours. Je viens demander à sainte Anne de l'aide pour apprendre à vivre avec mon infirmité. (Ouvrier de Montréal - 57 ans)

ou aide dans une situation difficile :

Je viens ici depuis l'âge de 12 ans. À cet âge-là, j'ai eu une jambe coupée dans un moulin à scie. Je me suis confié à sainte Anne; elle m'a beaucoup aidé et je suis venu la voir très souvent. (Beauceron - 80 ans)

Encore ici, il s'agit d'orienter la vie dans un sens positif. Et le courage ne consiste-t-il justement pas en cette capacité de vivre positivement une vie qui aurait de sérieuses raisons d'être vécue négativement? Le courage n'est-il pas une des formes les plus hautes de cette positivité que le pèlerin vient vivre à Sainte-Anne?

Encore une fois, cette positivité rejoint tout l'être et elle est liée à un approfondissement spirituel: «Je viens pour augmenter ma foi. C'est la foi qui me donne le courage de vivre. Je suis paralysé après être tombé d'une ligne à haute tension" (Monsieur de la Côte Nord, 60 ans).

Si on passe maintenant aux renseignements que nous fournissent les entrevues sur la sainte, on s'aperçoit que cette positivité, cette orientation vers un plus-être du cours de la vie est un trait de la relation avec la sainte. 
Ici encore cette positivité pourra prendre la forme du courage:

Sainte Anne m'a aidé constamment au cours des 20 dernières années. Lorsque mon mari est décédé, elle m'a donné le courage de reprendre goût à la vie. Je suis venu la voir bien souvent. (Dame de New-York - 55 ans)

du soutien :

J'ai fait ce long voyage poür venir la renconirer et ia prier. Ma famille m'en a parlé et je l'ai souvent priée au cours des heures sombres. Elle m'a soutenu toute ma vie et m'a donné le courage. (Comptable de Calgary - 46 ans)

du goût de vivre :

Quand mon mari est mort, j'étais seule et sans enfants. Je me suis découragée, puis j'ai crié comme une folle vers sainte Anne. J'ai alors senti comme une force qui me faisait reprendre goût à la vie. (Dame de Québec - 68 ans)

Et cette force, complémentaire du courage, appartient aussi à la relation du pèlerin à la sainte:

Dans ma vie, j'ai beaucoup souffert. Mon mari m'a abandonnée avec 4 enfants en bas âge. J'ai demandé à sainte Anne de m'aider et elle m'a fortifiée. Aujourd'hui, je puis dire que je suis heureuse et je viens ici tout exprès pour la remercier. (Dame des environs de Québec - 51 ans)

Mais ce que nous apprenons en plus, et déjà les exemples évoqués sont clairs à ce sujet, c'est que cette ouverture de l'être prend racine dans une relation suivie avec la sainte. On dira: «Je lui parle et elle me répond" (Confectionneur de Montréal); "elle m'a toujours aidé dans tout ce que je fais" (Jockey de Montréal); «c'est à elle que je confie mes soucis, mais je lui fais aussi part de toutes mes joies" (Dame d'Oshawa - 40 ans).

Cette relation de longue haleine est à la fois soutien du corps:

Ca fait longtemps que je devrais être morte. Sainte Anne me garde en vie; le coeur et l'épine dorsale sont irrémédiablement détériorés. C'est sainte-Anne qui me garde en vie et me donne la force de faire mon ouvrage. (Directrice d'un restaurant du NewJersey) 
et approfondissement du spirituel:

Nous venons chaque année... et nous découvrons d'année en année ce qu'elle peut faire. À chaque fois que nous venons, notre foi devient plus solide. Elle tient une très grande place dans notre vie. (Restaurateur de New-York - 49 ans)

La faveur ou ce qui est vécu comme le miracle est aussi le lieu privilégié d'une relation qui persiste. Combien diront: $\mathrm{j}$ 'ai été guéri, mon fils, ma mère, ma femme ont été guéris et nous venons souvent, à chaque année, la remercier et ce depuis 15, 20, 30 années... Une femme dont le mari qui avait été guéri d'une paralysie est décédé vient encore remercier comme elle le lui a promis au moment de sa mort. Dans d'autre cas, on n'a pu venir au moment de la guérison; mais on n'a pas oublié et on finira par venir, 20 ans plus tard s'il le faut. Dans d'autres cas, la continuité dans la relation apportera plusieurs faveurs étaliées dans le temps: «En 1967, j'ai été guérie d'un oeil malade; en 1979, j'ai été guérie d'un cancer du sein. Vous comprenez pourquoi je suis ici" (Dame du New-Hampshire).

La continuité, la persévérance dans la relation est donc source de grâce. S'il y a fidélité du pèlerin dans sa prière, il y a en réponse fícélité de la sainte envers ceux qui se réfêrent à elle.

\section{LES RÉCITS DE FAVEURS}

Les réponses de pèlerins nous suggèrent ces conclusions. Sontelles confirmées par le dossier des faveurs rapportées au sanctuaire pour ces mêmes années, c'est-à-dire de 1977 à 1982?

Quand on se met à la lecture du dossier des faveurs, une chose frappe: la fréquence des éléments qui suggèrent une relation de longue durée avec la sainte et à son lieu.

Nous pensons d'abord à ces cas où on vient rapporter l'événement bien après la guérison obtenue, à ces cas où un enfant guéri tout petit au lieu du pèlerinage ou à la suite d'une prière à sainte Anne revient ensuite, jeune adulte, remercier et rapporter sa guérison. Le nombre des années écoulées rendrait bien plausibles l'oubli, la négligence à poser le geste. Et pourtant on sent la mémoire, la ferveur de l'action de grâce encore bien vivantes. 
Plus significatifs encore sont les témoignages où l'accomplissement du pèlerinage et la guérison qui s'ensuit donnent lieu à un engagement de vie, voire à une conversion. Souvent, le miraculé deviendra organisateur de pèlerinages. Dans un autre cas, un indien d'Eskasoni venu faire son pèlerinage en chaise roulante a pu ensuite prendre contact avec un médecin qui lui a proposé l'opération qui l'a guéri. Il est venu remercier sainte Anne et s'est fait depuis catéchète dans sa réserve. Enfin, la guérison d'une personne peut susciter la conversion de son conjoint ou d'un proche. Ainsi l'épouse anglicane d'un américain guéri d'un cancer décide de se convertir au catholicisme et accomplit avec son mari les visites mensuelles auxquelles son mari s'était engagé.

La guérison donne donc lieu, outre une persistance à remercier, à un engagement spirituel et remarquons dans les exemples que nous venons de citer comment cet engagement reste lié au lieu comme à un ressourcement nécessaire du geste originel. Mais le cas le plus éclairant de cet engagement de vie qui prend sa source dans une guérison qui s'approfondit dans le temps et en relation avec le lieu qui lui a donné origine est celui-ci :

Une jeune fille de Philadelphie, qunique non-catholique a été guérie ici, il y a quelques années d'une maladie de coeur résultant d'une fièvre rhumatismale. Toutefois elle est restée très faible, mais elle est devenue infirmière. Pendant des années, elle est demeurée agressive et comme révoltée. Puis un jour, comme elle l'écrit, elle entendit comme une voix intérieure qui lui disait de devenir catholique. "Ce n'est pas ton corps qui doit guérir mais ton âme". Pendant neuf jours, elle vécut dans une agitation très grande, puis elle fit les premières démarches et finalement se convertit. "Je suis venue à Ste-Anne pour remercier»; elle est convaincue que cette grâce lui a été obtenue par un des malades qui se trouvait ici. "Je réalise que la plus grande grâce que Dieu peut nous accorder, c'est d'être unie à Lui, dans la parfaite conformité à sa volonté. Je suis une pauvre âme qui ne mérite pas tout ce qui m'a été accordé et je m'abandonne entièrement à la volonté de Dieu». «Quand j'ai revu Ste-Anne, j'ai vu à nouveau la joie et la paix qu'il y avait sur le visage des malades qui étaient ici. Je suis en bonne santé maintenant, mais je veux vivre avec l'état d'âme de ces handicapés qui sont tournés vers le Seigneur».

Dans ce cas, la guérison est l'événement de départ d'une suite qui devait mener la personne à un changement d'attitude radical dans sa vie spirituelle. D'ailleurs, s'il faut prendre le texte dans la spontanéité de sa rédaction, la santé de la jeune femme, de très faible qu'elle était au moment de la guérison, est dite bonne à la fin du récit. L'évé- 
nement guérisseur se situe donc au point de départ d'une évolution de toute la personne. Une guérison n'est pas un événement ponctuel. C'est un fait qui a des résonances lointaines, profondes et qui atteint toute la vie.

Quelques autres cas nous paraissent aussi intéressants à citer pour illustrer les formes liées à ce changement de vie qui prend son origine dans l'obtention d'une faveur à Ste-Anne.

Le premier de ces exemples se pose, à l'inverse du premier, comme une non-guérison. Et cette non-guérison ou plutôt la décision d'assumer cet "échec" dans la demande a orienté cette personne vers ce qui sera nommé dans le texte «une vie nouvelle». De quoi s'agit-il? Une américaine dont la soeur alors âgée de 12 ans et très malade avait été guérie en 1940 vient à Ste-Anne en 1979 affectée de sclérose. À la suite de son pèlerinage, elle comprend qu'elle ne doit pas attendre une guérison et organise avec son mari un groupe de Renouement conjugal pour handicapés. Ils trouvent là un point de départ à une "vie nouvelle» et viennent remercier à Ste-Anne pour cette nouvelle orientation de leur vie.

Enfin, un dernier cas où le changement précède plutôt la guérison qu'il ne ia suit, où la faveur réside précisément dans cette décision de changement qui ouvrira la voie vers un retour à la santé, un nouvel engagement de vie et un approfondissement de la foi :

En mars 79, un jeune étudiant en droit du Connecticut vint en pèlerinage au sanctuaire. Il était en dépression nerveuse, à la suite de poussées d'arthrite qui lui déformaient les mains et lui causaient des maux de tête intolérables. Il avait de plus des ulcères à l'estomac. Sur le conseil de sa famille et de son médecin, il fit le pèlerinage à Ste-Anne de Beaupré. Il demanda à sainte Anne de lui indiquer quelle était sa vraie vocation dans la vie; depuis plusieurs semaines, il était hanté par la pensée du suicide. Il lui vint alors à l'esprit qu'il devait entreprendre une carrière d'artiste. Un mois plus tard, les ulcères étaient guéris et l'arthrite était disparue des mains; les douleurs arthritiques disparurent exactement le même jour où il prit ses premiers cours de dessin. Après trois mois de cours, il fut choisi comme un des espoirs artistiques et admis à la Société Internationale des Peintres de la Marine. Il est maintenant reconnu comme un des peintres importants de cette catégorie mais veut se consacrer à la peinture religieuse. Après son pèlerinage il est revenu à la foi catholique et est entré dans le Tiers Ordre franciscain. 
On peut sourire ici devant la limpidité des faits; là n'est pas ce qui nous intéresse. Voici un cas où le pèlerinage a été à l'origine d'une réorientation complète de la vie dont ont découlé à la fois santé et retour à une vie spirituelle plus intense.

Les cas que nous venons de citer, où une faveur obtenue au pèlerinage (guérison, courage d'assumer positivement son handicap, reconnaissance de ses besoins profonds) détermine un nouvel engagement de vie, un renouveau de tout l'être, font du pèlerinage et de la faveur un énément qui a des répercussions sur toute la vie.

Le deuxième trait que nous révèle le dossier des faveurs concernant la double relation à la sainte et à son lieu tient à l'engagement qui s'y manifeste. La dimension temporelle, l'insertion de la faveur dans la durée de la vie y sont encore présentes; mais s'y ajoutent un engagement envers sainte Anne, engagement qui souvent pourra passer par son lieu.

Une première forme de cette relation où l'engagement apparaît très clairement se trouve dans une série d'exemples où l'engagement envers la sainte précède l'obtention de la faveur: la guérison se trouve alors liée à cette forme de relation qui s'attache à la personne, qui mise sur elle.

Premier cas de cette série: celui d'une dame guérie d'un cancer du pancréas. Elle a fait d'abord une série de neuf pèlerinages à Sainte-Anne pendant neuf dimanches de suite. Mais l'événement qui détermine la guérison est la décision, alors qu'une crise qui la mène habituellement à l'hôpital s'annonce, de s'en remettre à une prière à sainte Anne. Une semaine plus tard, son médecin confirme qu'il n'y a plus de trace de cancer. Ici, il y a eu engagement de la personne envers la sainte, engagement préparé sans doute par la série des neuf pèlerinages et suivi, il va sans dire, de plusieurs autres.

Cet engagement déterminant de la personne avant l'obtention d'une faveur peut se retrouver ailleurs. Ainsi le cas de cette femme, une égyptienne de Montréal, à qui les médecins conseillaient l'avortement parce que l'enfant qu'elle portait ne devait pas être normal, qui vient plutôt recommander son enfant à Ste-Anne. Il naîtra en parfaite santé et l'heureuse mère viendra rapporter le fait au moment d'un pèlerinage d'action de grâces. Ici, le pèlerinage vient soutenir un engagement de vie préalable; comme dans le cas précédent, on mise sur la sainte, sur la dévotion exprimée à son lieu. Le pèlerinage cristallise l'engagement 
de la personne: cette femme refuse la solution médicale mais fait une démarche pour orienter la vie dans un sens positif.

Évoquons enfin un exemple où les démarches répétées au lieu du pèlerinage, auxquelles répondent d'ailleurs les éléments d'un retour progressif à la santé, accomplissent l'engagement envers la sainte. Une dame de Montréal vient pendant douze semaines en pèlerinage à SteAnne recommander son fils policier abattu dans un hold-up. Au moment où elle décide de venir, il est entré dans un demi-coma et on attend sa mort d'heure en heure. À chacune des visites il prend du mieux jusqu'à ce que les médecins le déclarent hors de danger. Soulignons le remarquable parallélisme du récit entre la multiplication des visites au sanctuaire, l'engagement dans la démarche pèlerine et le retour progressif à la santé.

Trois cas donc où à des degrés divers on retrouve ces éléments: échec des moyens humains pour assurer un retour à la santé; approfondissement d'une relation avec la sainte et à son lieu qui se marque soit par le risque pris en misant sur elle, par le choix qu'on fait d'elle au détriment de tout autre, soit par la longue série des démarches entreprises à son lieu. Le pèlerinage et la dévotion sont donc l'objet d'un approfondissement qui se marque à la fois dans le temps et dans la mobilisation de la personne qu'il suppose envers la sainte.

Autre forme d'engagement envers la sainte: le voeu. L'engagement précède encore la guérison mais cette fois, il est sous condition; le voeu ne sera accompli qu'après la guérison. Malgré cette restriction que nous ne pouvons marquer de faire et qui met une sourdine au risque de l'engagement vis-à-vis la personne sainte contenu dans le voeu, nous croyons que, malgré son mode conditionnel, le voeu constitue un engagement très réel vis-à-vis la personne sainte à cause de la durée souvent très longue qu'il introduit dans la relation ou du sacrifice qu'il suppose. Celui qui fait un voeu se lie à un "service" de la sainte pour une bonne partie de sa vie et souvent même, les exemples vont nous le montrer, cette relation va persister bien au delà du cadre contractuel du voeu.

Citons par exemple le cas de cet homme qui en 1940 se mourait des suites d'une pleurésie et qui, après avoir promis d'aller pendant 25 ans au pèlerinage le jour de la fête, sent le besoin de s'en aller tout de suite travailler. Quarante-deux ans après il rapporte n'avoir manqué son pèlerinage qu'une fois, l'année du décès de sa femme au mois de juillet. Ou encore cet indien de Bersimis affecté d'une pneumonie 
et que le médecin veut renvoyer mourir dans sa famille, qui promet de s'abstenir d'aller à la chasse pendant dix ans. L'objet de la promesse ne concerne pas directement sainte Anne mais ce sacrifice ne se veut-il pas un hommage à celle dont on implore la guérison? De toute manière, le lien durable existe bien puisqu'il affirme ne jamais manquer sa "sainte Anne». Enfin, c'est dans cette perspective de l'établissement d'un lien de longue durée qu'il nous faut comprendre, croyons-nous, cette coutume d'abonner quelqu'un à vie à la revue à la suite d'une guérison, ou de promettre de le faire.

Puisque nous parlons d'engagement envers la sainte, je pense qu'il faut aussi signaler des témoignages où tout un groupe s'engage soit dans une prière répétée qui aboutit à une guérison, soit dans une promesse qui le lit à sainte Anne dans la durée.

Ainsi, ce texte savoureux et imaginé, dont les aspects pittoresques ne doivent pas masquer la rigueur de l'engagement pris: chaque membre de la famille a fait voeu d'un pèlerinage annuel à Ste-Anne et celui qui rapporte l'événement le fait encore 25 ans après, bien qu'il s'agisse d'un engagement pris alors qu'il était enfant:

Ce fait a été raconté en mai dernier (1981), mais fait allusion à des événements qui datent de 1956 . Un jeune homme est venu au bureau et il a expliqué pourquoi dans sa famille on avait une grande dévotion à sainte Anne. "C'est à cause de ma petite soeur; toute la famille s'est engagée par voeu, individuellement à venir faire un pèlerinage annuellement ici à Sainte-Anne. À date, personne n'y a manqué. La petite soeur était née avec une défectuosité du tube digestif; une partie de ce tube n'était pas formée et elle ne pouvait rien avaler. Pendant une année entière, après sa naissance la petite fille a été hospitalisée; on la nourrissait artificiellement, mais elle ne tenait plus à la vie que par un fil. C'est alors que la famille a fait son voeu. On est venu ensemble en pèlerinage de Montréal à Québec. Le soir, au retour du pèlerinage, on était attablé pour le repas du soir; au menu, il y avait du porc, qu'on venait de faire cuire. La maman, après avoir servi la famille, prit la petite fille sur elle et se mit à souper. Tout à coup, l'enfant s'échappe de ses bras et prend "une poignée de porc frais" et l'avale alors qu'elle n'avait jamais avalé de sa vie. On téléphona au médecin qui conseilla de la veiller toute la nuit parce qu'elle en mourrait. Elle dormit à poings fermés et le lendemain elle se réveilla en parfaite condition. Elle n'a jamais eu aucun problème depuis ce soir-là. 
Il s'agit dans ce cas d'un engagement de toute la famille, et dans la longue durée faut-il le dire, engagement entériné par un pèlerinage et dont l'objet consiste en une suite de pèlerinages. Ailleurs on aura tout simplement recours à une prière répétée à sainte Anne. On rapporte ainsi le cas d'un bébé de Montmagny.

opéré le jour de la fête de sainte Anne 1976 pour une tumeur au cerveau, à l'âge de six mois. C'était une tumeur qui était considérée comme "reproductive" c'est-à-dire que selon ce qu'a dit la mère, on avait beau l'inciser, elle se développait toujours. On n'a pu enlever que la moitié de la tumeur, de la grosseur d'un oeuf. Après l'opération, le médecin a dit: «Il en a pour deux semaines à vivre». Mais toute la famille et spécialement ses deux petits frères l'ont recommandé tous les jours à sainte Anne. Après quatre mois, non seulement il n'était pas mort, mais semblait de plus en plus vigoureux: il marchait, commençait à parler etc. On prit une radiographie et il ne reste absolument aucune trace de la tumeur. Le docteur a dit à la mère : «Ce n'est pas moi qui ai enlevé ce qui restait de la tumeur... vous avez dû recourir à une prière spéciale».

Le récit se fait tout simple ici: une famille prie pour le plus petit de ses membres quotidiennement, pendant au moins quatre mois et on souligne, sans doute comme particulièrement efficace dans sa candeur innocente et peut-être aussi dans sa non-nécessité, la prière des petits frères. Une famille a persisté dans sa prière à sainte Anne et cette durée a ouvert une voie qu'on croyait fermée. Ailleurs c'est une communauté paroissiale tout entière qui obtiendra la guérison d'un de ses membres et viendra effectuer, organisé par le conjoint, un pèlerinage de reconnaissance. Ailleurs, c'est le pèlerinage du beau-père accompagné de deux de ses enfants qui voudra à un homme de retrouver l'usage de la parole et de l'écriture perdu à la suite de la désintégration de cellules du cerveau. L'engagement vis-à-vis la sainte par la prière, par le pèlerinage est ici vécu par une collectivité pour un de ses membres.

Que faut-il retenir finalement du point de vue qu'apportent les faveurs à notre dossier? Nous avons tout d'abord souligné la double insertion de la faveur dans une durée relationnelle avec la sainte: durée subséquente à l'obtention d'une faveur, la faveur est le lieu d'une relation qui persiste; durée antécédente à l'obtention d'une faveur: la persistance dans la relation avec la figure sainte est source de grâces. La faveur dénote souvent aussi un engagement vis-à-vis la personne sainte: face à l'échec des recours humains ou face à l'objection de conscience devant les solutions proposées comme dans le cas du refus de l'avortement que nous avons cité, on mise sur la personne sainte, 
on «met sa foi» en elle, abandonnant les autres voies de solution. La faveur dénote donc essentiellement un mode de vie relationnel, mode de vie qui implique intégration de cette relation avec une figure sainte dans la vie de l'homme à la fois dans une durée et dans une ouverture qui peut aller jusqu'à l'engagement: la personne sainte est alors celle à qui on donne sa foi, à qui on se confie, dont on attend la protection. Par cette relation qui s'étend dans la durée avec une personne sainte, par l'engagement qu'elle peut amener envers elle, la faveur devient le lieu d'une possivité qui a des incidences et des ramifications spirituelles profondes: elle participe donc ainsi aux caractères mêmes de la prière et de la démarche pèlerine à Ste-Anne.

Puisque nous avons maintenant passé en revue nos trois types de témoignage pèlerin pour voir ce qu'ils avaient à nous apprendre à la fois sur la personne sainte et sur le lieu de son pèlerinage, que pouvons-nous en conclure sur la relation personne sainte-lieu? Comment se vit cette intégration au point de départ contradictoire entre une personne sainte, figure universelle de sainteté et de recours, et un lieu bien délimité dans ses coordonnées spatiales?

Que nous disent nos documents, prières des feuillets, entrevues, témoignages de faveurs rapportés au bureau du pèlerinage? Tous ces documents avec leurs insistances propres nous parlent d'une relation avec une sainte. Les comportements qu'ils évoquent ont tous les traits de comportements relationnels: emploi d'un langage aux résonances affectives; expression d'une confiance où on donne sa "foi " à une personne et où on attend d'elle, en retour, une aide; affection et confiance donc envers une personne qu'on nomme mère ou grandmère ou plutôt qu'on considère dans sa double maternité au premier et au second degré à la fois objective, par les événements qui ont marqué sa vie terrestre, et subjective; présence enfin de cette mère dans sa vie qui devient mode de vie, dans une relation maintenue dans la durée et dans un engagement qui, face à la limite, privilégie cette relation personnelle, mise sur la personne sainte. Nous trouvons donc le vocabulaire de la relation, d'une relation à laquelle on recourt pour surmonter la limite, qui soutient le cheminement vers un plus être de l'homme, d'une relation dont l'approfondissement ouvre sur la positivité de la vie.

Mais ce qui est essentiellement vécu comme une relation appelle toujours en complément le lieu. Qu'il s'agisse de prière déposée au pied de la statue, d'accomplissement ou de promesse de pèlerinage, d'un récit de faveur rapporté au bureau du pèlerinage, le lieu entre 
comme de soi à l'intérieur de cette relation. Si on est guéri sans intervention du lieu on gardera, nous avons vu jusqu'à quel point, désir d'accomplir au lieu du pèlerinage son action de grâces. En fait, la relation que nous avons relevée plus haut est double dans cette dévotion: il y a à la fois relation à la personne sainte et au lieu. La relation à la personne sainte prend figure de la relation au lieu. L'adéquation est tellement forte qu'on peut presque dire qu'il s'agit d'une relation avec une personne sainte qui est un lieu. Essentiellement le lieu donne la présence de la figure sainte. "Si vous saviez comme c'est merveilleux de se retrouver près d'elle" (Dame de Charny - 40 ans). "Ma mère a toujours eu une grande dévotion à sainte Anne. Quand j'étais petit, je venais avec elle à Sainte-Anne de Beaupré, tous les deux ans. Cela m'a marqué en profondeur. La bonne sainte Anne a gardé une grande place dans mon coeur et je me suis mis à affectionner beaucoup l'endroit. Je reviendrai bien des fois" (Armateur du Michigan - 51 ans), dans une réponse où, de la venue au lieu découle la dévotion et de la dévotion, l'amour du lieu; on circule ici en toute liberté de l'un à l'autre. "Sainte Anne est partout ici" (Dame de Québec), dans une formulation où l'adéquation est parfaite entre la sainte et le lieu. "C'est le lieu où sainte Anne nous attend" (Directeur, de Québec) «Je suis venu cette année, avec le pressentiment que sainte Anne m'aimait; c'était comme si elle m'appelait personnellement à venir à Beaupré. Depuis que je suis ici, je ressens une sorte de chaleur intérieure qui me surprend" (Cheminot de Montréal - 55 ans), où la sainte dans une relation toute personnelle appelle au lieu. La relation avec la personne sainte sous-tend donc une relation avec un lieu.

Mais pourquoi venir à ce lieu? Que s'y passe-t-il qui appelle le retour? Quelle réalité ont évoquée nos témoins au sujet du lieu? Ils nous ont parlé d'un lieu où on se sent à l'aise, comme chez soi, d'un lieu où on est bien, d'un lieu qui abolit les barrières sociales et les différences raciales. Ici on trouve la paix, ici on goûte le silence et l'arrêt du bourdonnement des préoccupations habituelles. Ici encore on retrouve une capacité de prier à son goût, tout son saoul, et cette prière jaillit comme de soi et sans effort. Ici la guérison peut 
même s'accomplir sans qu'on ait besoin de la demander ${ }^{10}$, au simple contact avec le lieu ${ }^{11}$. C'est au lieu que l'homme est unifié dans une participation à la fois physique et spirituelle de son être, dans une guérison du corps qui passe par les voies d'une ouverture au spirituel, dans un engagement spirituel qui passe par les voies d'une démarche corporelle, venue au lieu, marche d'une procession, ascension à genoux de la Scala Santa, etc. Ici la prière s'agrandit aux dimensions du monde, unifiant la foule pèlerine en un peuple. Le lieu accomplit donc ce qu'on vient y chercher: de soi il nous établit dans une réalité de bien-être, de paix, de santé, de prière. Il est efficace et accomplit déjà chez le pèlerin les traits d'une ouverture au plus-être sous l'une ou l'autre de ses formes. Ainsi, à cette femme souffrant de dépression il parle d'espérance et accomplit en elle cette recharge vitale qui lui permet de retrouver une vision positive des choses: "Pour moi, l'église de Sainte-Anne, c'est un coin d'espérance; c'est ici que je suis parvenue à me sortir de ma dépression chronique. Ce n'est pas tellement l'édifice qui m'intéresse, mais c'est qu'ici tout me parle d'espérance" (Dame du Bas du Fleuve 55 ans). Le lieu accomplit certaines réalités et c'est pourquoi il faut $y$ revenir périodiquement.

À reprendre ainsi que nous venons de le faire tous les caractères du lieu, on en conclut vite qu'il s'agit d'un lieu qui est plus qu'un lieu. En fait, de l'aveu même des pèlerins, ce lieu est celui de tout autre:

Je conseillerais de venir ici parce que c'est un lieu complètement différent de tout ce qu'on trouve ailleurs. Ce n'est pas facile à exprimer en des mots, mais on a l'impression de toucher le «tout autre». C'est un lieu privilégié... différent des lieux ordinaires. (Dame de Québec - 75 ans) et une jeune handicapée me disait,

\footnotetext{
${ }^{10}$ Une dame de Détroit était restée infirme, par suite d'un accident d'automobile survenu en 1967. Le cou était dévié sur le côté et l'épaule était très douloureuse. Elle est venue à Ste-Anne en 1981 et est allée prier au pied de la grande statue aux intentions d'un de ses enfants. Tout à coup elle a eu l'impression qu'elle était comme agenouillée sur des petites pierres; c'était très douloureux mais elle a pu tenir le coup pendant cinq minutes. En sortant de l'église elle s'aperçoit qu'elle n'a plus de douleurs et que son cou est redevenu normal.

11 «Une Israélite de New-York nouvellement convertie, vient en pèlerinage au début de juillet. Elle avait le désir d'entrer en communauté, mais elle était toujours refusée, par suite d'une infirmité chronique. Elle souffrait d'un défaut de la colonne vertébrale qui l'obligeait à toujours garder la tête levée vers le haut. Elle ne pouvait aucunement pencher la tête ou la tourner à gauche ou à droite. Elle vient en pèlerinage à SainteAnne et en descendant de l'autobus, sa tête reprend la place qu'elle devait avoir normalement".
} 
opposant au caractère partiel des vacances habituelles où on ne peut faire qu'une chose à la fois, nager, pêcher, la plénitude de ce lieu: Here it's everything!"

Le contact avec ce tout autre rend tout autre et c'est en cela d'ailleurs que le lieu accomplit l'ouverture à ce qu'on vient y chercher: "Ce n'est pas tellement la Basilique qui m'impressionne, mais c'est le fait d'être dedans... d'être là en personne. On se sent tout autre». (Fonctionnaire de Montréal - 55 ans).

Le lieu accomplit de soi l'ouverture au positif recherchée parce qu'il ouvre au tout autre, à une totalité qui prend l'homme dans son entier et le change. Le lieu apparaît donc plus que le lieu. Si nous avons pu énoncer une première proposition: une dévotion centrée sur un pèlerinage est une relation avec une personne sainte qui est un lieu, nous pouvons maintenant y ajouter un complément: ce lieu nous renvoie hors du lieu, à plus que lui. Nous pouvons reconnaitre les dimensions eschatologiques auxquelles il réfere: ce qui est vécu là est un commencement de ce qui sera vécu dans l'au-delà: "cette église est le symbole et le signe de ce qui nous attend en haut", dira un commerçant de Loretteville. À leur manière les chants reconnaissent cette continuité: «Bonheur dans la vie devant votre autel; et dans la patrie, bonheur éternel!» ${ }^{12}$

Nous avons nommé lieu symbolique cette réalité du lieu qui nous renvoie au-delà de lui. Que disent nos documents sur ce lieu qui nous renvoie hors de lui et que nous nommons lieu symbolique? Ils nous disent d'abord que le pèlerin vit en ce lieu une relation où la prière ouvre sur un sens positif de la vie, lieu une relation où la prière ouvre un sens positif de la vie, "santé, pardon, grâce et bonheur", trouve$\mathrm{t}$-on dans le traditionnel et beau chant $\mathrm{du}$ "Vive Sainte Anne», sur un renouveau de la personne. Cette relation de présence engage la durée et devient mode de vie. Ici l'homme est unifié corps et âme dans un renouveau du sens positif de la vie, qu'il s'agisse d'une guérison, d'un renouveau du courage, d'une conversion, d'un dynamisme nouveau pour changer sa vie ou plus simplement encore de la capacité réalisée comme spontanément de prier à "son goût". Ici l'amélioration d'un état du corps ou la capacité de vivre positivement une situation difficile liée à des déficiences de celui-ci ou à des circonstances émotivement éprouvantes, la capacité d'orienter sa vie plus positivement sont vécues dans une ouverture au spirituel qui s'exprime dans une relation avec une personne sainte dont la présence se vit dans un rapport à un lieu qui est le sien

${ }^{12}$ Vers son sanctuaire, couplet 10. 
et élu par elle et par Dieu comme lieu de sa présence et des manifestations de sa bonté. C'est probablement par son lien à un lieu, par cette dimension spatiale attachée à sa personne que la figure sainte atteint tout l'être physique et spirituel du pèlerin qui ira vers elle et dont l'émerveillement de trouver là une capacité nouvelle de prier traduira l'intégration de son être physique et spirituel.

Terminons donc par une tentative de définition de ce que nous avons nommé le lieu symbolique, en tenant compte des réalités que nous venons d'évoquer mais aussi d'autres facettes du pèlerinage dont il ne nous était pas loisible de parler aujourd'hui. Le pèlerinage fournit un lieu symbolique parce que l'implantation cosmique, l'insertion temporelle dans une histoire réelle ou projetée dans un légendaire des origines, la figure sainte, l'iconographie, la prière dite ou changée, le gestuel mettent en branle une certaine vision du monde totale, globale et unifiante d'un vécu. Dans un lieu symbolique, l'homme pèlerin vit une représentation du monde à laquelle il participe, qui redonne sens à la vie en l'ouvrant à la positivité, une représentation du monde qui unifie l'homme dans une conjugaison de l'expression physique et spirituelle de son être. De Ste-Anne de Beaupré le pèlerin sort plus un et cette expérience "heureuse" d'unification de son être a portée d'un commencement d'au-delà.

Anne DORAN-JACQUES

Chicoutimi 